\title{
Prevalence of Micronutrient Deficiency after Bariatric Surgery
}

\author{
Eva-Christina Krzizek ${ }^{a, b}$ Johanna Maria Brix ${ }^{a, b} \quad$ Alexander Stöckl ${ }^{a, b}$ \\ Verena Parzer ${ }^{a}$ b Bernhard Ludvik ${ }^{a, b}$ \\ ${ }^{a}$ Department of Medicine I, Hospital Landstrasse, Vienna, Austria; ${ }^{b}$ Karl Landsteiner Institute of Obesity and \\ Metabolic Diseases, Vienna, Austria
}

\section{Keywords}

Micronutrients · Vitamins · Bariatric surgery · Sleeve

gastrectomy $\cdot$ Roux-en-Y-gastric bypass

\begin{abstract}
Introduction: While vitamin deficiency after bariatric surgery has been repeatedly described, few studies have focused on adequate micronutrient status. In this study, we examine the prevalence of vitamin and micronutrient deficiency for the first 3 years after surgery. Methods: Out of 1,216 patients undergoing surgery, 485 who underwent postoperative follow-up in an outpatient clinic between 2010 and 2019 were included in this evaluation $(76.9 \%$ women, mean age $42 \pm 12$ years, mean BMl: year 1, 33.9 \pm 19.2 ; year 2, $29.7 \pm 8.7$; year 3, $26.2 \pm 4.0$ ). Weight and cardiovascular risk factors as well as ferritin, vitamin B12, folic acid, 25-OH-vitamin D, vitamin A, vitamin E, zinc, copper, and selenium were evaluated. Deficits were defined as follows: ferritin $<15 \mu \mathrm{g} / \mathrm{L}$, vitamin B12 $<197 \mathrm{pg} / \mathrm{mL}$, folic acid $<4.4 \mathrm{ng} /$ $\mathrm{mL}, 25-\mathrm{OH}$-vitamin $\mathrm{D}<75 \mathrm{nmol} / \mathrm{L}$, vitamin $\mathrm{A}<1.05 \mu \mathrm{mol} / \mathrm{L}$, vitamin $\mathrm{E}<12 \mu \mathrm{mol} / \mathrm{L}$, zinc $<0.54 \mathrm{mg} / \mathrm{L}$, copper $<0.81 \mathrm{mg} / \mathrm{L}$, and selenium $<50 \mu \mathrm{g} / \mathrm{L}$. All patients underwent dietary counselling and substitution of the respective deficits as appropriate. Results: One year after bariatric surgery, 485 patients completed follow-up. This number decreased to 114 patients in year 2 , and 80 patients in year 3 . Overall, $42.7 \%(n=$
\end{abstract}

karger@karger.com www.karger.com/ofa

Karger"

BOPEN ACCESS
(C) 2021 The Author(s)

Published by S. Karger AG, Basel

This is an Open Access article licensed under the Creative Commons Attribution-NonCommercial-4.0 International License (CC BY-NC) (http://www.karger.com/Services/OpenAccessLicense), applicable to the online version of the article only. Usage and distribution for commercial purposes requires written permission.
207) underwent sleeve gastrectomy, $43.7 \%(n=211)$ Rouxen-Y-gastric bypass, and $13.9 \%(n=67)$ gastric banding. The following deficits were found (year 1/2/3): ferritin, 21.6/35.0/32.5\%; vitamin B12, 14.3/1.8/6.3\%; folic acid, 29.7/21.6/15.3\%; 25-OH-vitamin D, 70.8/67.0/57.4\%; vitamin A, 13.2/8.9/12.8\%; vitamin E, 0\%; zinc, 1.7/0/1.5\%; copper, 10.4/12.2/11.9\%; selenium, 11.1/4.3/0\%. Conclusion: As seen in other studies, the follow-up frequency decreased over the years. Despite intensive substitution, the extent of some deficiencies increased or did not improve. These results suggest reinforcing measures to motivate patients for regular follow-up visits, considering closer monitoring schedules, and improving supplementation strategies.

(c) 2021 The Author(s)

Published by S. Karger AG, Basel

\section{Introduction}

Obesity is becoming a worldwide pandemic disease and is recognized as a major health concern by the WHO. Data showed a prevalence of $39 \%$ for overweight and $13 \%$ for obesity worldwide in 2016, values which have tripled since 1975 [1].

The number of bariatric procedures as an effective treatment for morbid obesity is rising steadily. Although postoperative micronutrient deficiency is a well-known side effect of bariatric surgery [2-4], probably due to al- 
Table 1. Vitamin deficiency before surgery, 1, 2, and 3 years after surgery, and reference values for micronutrients

\begin{tabular}{lcccc}
\hline & Baseline, \% & Year 1, \% & Year 2, \% & Year 3, \% \\
\hline Ferritin $(15-150 \mu \mathrm{g} / \mathrm{L})$ & 8.3 & 21.6 & 35.0 & 32.5 \\
Hemoglobin $(>12.5 />13 \mathrm{~g} / \mathrm{dL})$ & 15.1 & 28.2 & 36.4 & 37.0 \\
Vitamin B12 $(188-771 \mathrm{pg} / \mathrm{mL})$ & 4.8 & 14.3 & 1.8 & 6.3 \\
Folic acid $(5.3-18.7 \mathrm{ng} / \mathrm{mL})$ & 62.4 & 29.7 & 21.6 & 15.3 \\
25-OH-vitamin D $(>75 \mathrm{nmol} / \mathrm{L})$ & 93.9 & 70.8 & 67.0 & 57.4 \\
Vitamin A $(1.05-2.45 \mu \mathrm{mol} / \mathrm{L})$ & 5.7 & 13.2 & 8.9 & 12.8 \\
Vitamin E $(11.6-46.4 \mu \mathrm{mol} / \mathrm{L})$ & 0 & 0 & 0 & 0 \\
Copper $(0.81-1.94 \mathrm{mg} / \mathrm{L})$ & 4.4 & 10.4 & 12.2 & 11.9 \\
Zinc $(0.54-1.03 \mathrm{mg} / \mathrm{L})$ & 1.0 & 1.7 & 0 & 1.5 \\
Selenium $(50-120 \mu \mathrm{g} / \mathrm{L})$ & 0 & 11.1 & 4.3 & 0 \\
\hline
\end{tabular}

tered eating habits and the restrictive/malabsorptive component of the operation, most studies concentrate on only the evaluation of a few micronutrients in smaller cohorts (e.g., vitamin D, vitamin B12, ferritin). Following bariatric surgery, important sites of absorption of various minerals and vitamins such as the duodenum and the proximal jejunum might be excluded from absorption. Therefore, life-long follow-up and appropriate substitution of macro- and micronutrient deficiencies is crucial following bariatric surgery. However, only $12-49 \%$ of patients have an assessment of micronutrient status after the procedure - depending on the kind of micronutrient [5]. A nationwide study in France also showed that the frequency of visits to the doctor and the intake of supplements decreased over the years [6]. In this study, we examined the prevalence of micronutrient deficiency in a large cohort of patients after bariatric surgery in the longterm follow-up, and analyzed a greater number of possible deficiencies than usually regarded in order to evaluate the need for nutritional counselling and appropriate postoperative substitution.

\section{Research Design and Methods}

For recruitment, patients with morbid obesity who underwent a postoperative check-up after bariatric surgery were enrolled at the obesity clinic of the 1st Department of Medicine at the Hospital Landstrasse, Vienna, Austria between 2010 and 2019. Patients were referred by their general practitioner or the associated surgery department for postoperative evaluation. The study was approved by the institutional Ethics Committee (Ethics Committee of "Magistrat der Stadt Wien MA 15"; EK 19-077-VK) and complied with the Declaration of Helsinki, including current revisions [7], and the Good Clinical Practice guidelines. All subjects gave written informed consent before the beginning of the study. All patients were treated by an interdisciplinary team of surgeons, endocrinologists, dietitians and, if necessary, psychologists.
Four hundred and eighty-five patients following bariatric surgery $(76.9 \%$ women, age $42 \pm 12$ years, mean BMI $33.9 \pm 19.2)$ were available after the first year of surgery and were investigated in this cross-sectional, retrospective study. During the following years, the rate of participants decreased steadily. All patients underwent close nutritional counseling by a trained dietitian directly after surgery, after 6 months, and at least once a year. In patients with deficiencies, dietary follow-ups were performed more frequently on an individual basis. Depending on their vitamin status, patients received one or more supplements in addition to the standard supplementation and were regularly instructed to adjust the dose accordingly.

Starting after surgery, all patients were advised to take a standard supplementation with vitamin D (2,200 IU/day), calcium (1,200 $\mathrm{mg}$ /day), folic acid ( $\mathrm{mg} /$ day), a multivitamin preparation, and, in case of Roux-en-Y-gastric bypass (RYGB), hydroxocobalamin ( $1 \mathrm{mg}$ every 3 months). At our clinic no standard iron supplementation is performed. Even women after RYGB are supplemented accordingly to their laboratory results.

Anthropometric data (such as height, weight, and BMI) were assessed. Additionally, cardiovascular risk factors such as blood pressure and parameters of glucose and lipid metabolism were recorded. Blood samples were taken after an overnight fast and immediately processed by the hospital's laboratory.

\section{Laboratory Measurements}

Blood glucose, total cholesterol, high-density lipoprotein cholesterol, and triglyceride levels were measured by enzymatic in vitro tests (Roche Diagnostics GmbH, Graz, Austria). Low-density lipoprotein cholesterol was calculated using the Friedewald formula. Glycosylated hemoglobin (HbA1c) was measured by HPLC (Diamat; Bio-Rad Laboratories, Hercules, CA, USA). Hemoglobin was measured by the SLS (sodium lauryl sulfate)-detection method (Sysmex, Austria).

Vitamin A as well as vitamin E levels were measured by the HPLC method. Vitamin B12, 25-OH-vitamin D, parathyroid hormone (PTH), and folic acid were determined by electrochemiluminescence assay (Cobas e immunoassay system, Roche Diagnostics, Germany). Ferritin was measured by quantitative in vitro tests (Roche/Hitachi Cobas e system, Germany). Zinc, copper, and selenium were measured via atomic absorption spectrometry with the "Graphitrohrofen technique." For the reference range of the respective laboratory tests, see Table 1. 
Table 2. Baseline characteristics (mean \pm $\mathrm{SD})$

\begin{tabular}{lcccc}
\hline & Baseline & Year 1 & Year 2 & Year 3 \\
\hline Age, years & $41 \pm 12$ & $42 \pm 10$ & & \\
Weight, kg & $128 \pm 24$ & $92 \pm 32$ & $85 \pm 20$ & $85 \pm 21$ \\
BMI & $44 \pm 9$ & $34 \pm 19$ & $29 \pm 8$ & $26 \pm 4$ \\
HbA1c, \% & $6.1 \pm 2.5$ & $5.5 \pm 2.3$ & $5.4 \pm 0.5$ & $5.5 \pm 0.8$ \\
Systolic blood pressure, mm Hg & $141 \pm 17$ & $126 \pm 17$ & $122 \pm 18$ & $123 \pm 13$ \\
Diastolic blood pressure, mm Hg & $90 \pm 11$ & $80 \pm 11$ & $77 \pm 11$ & $80 \pm 12$ \\
Cholesterol, mg/dL & $203 \pm 205$ & $228 \pm 603$ & $224 \pm 425$ & $177 \pm 34$ \\
High-density lipoprotein cholesterol, & $47 \pm 14$ & $58 \pm 15$ & $63 \pm 16$ & $66 \pm 15$ \\
$\quad$ mg/dL & $118 \pm 34$ & $105 \pm 35$ & $99 \pm 34$ & $91 \pm 28$ \\
Low-density lipoprotein cholesterol, & & & & \\
$\quad$ mg/dL & $163 \pm 101$ & $99 \pm 52$ & $99 \pm 45$ & $99 \pm 45$ \\
Triglyzerides, mg/dL & & & & \\
\hline
\end{tabular}

Statistics

Metric data are presented as the mean (SD) or as the median (IQR) as appropriate, while categorial variables are presented as absolute numbers and relative percentages. The laboratory values of the micronutrients are dichotomized according to local laboratory reference ranges. Hypovitaminosis $\mathrm{D}$ is defined by $25-\mathrm{OH}-$ vitamin $\mathrm{D}$ values below $75 \mathrm{nmol} / \mathrm{L}$ (insufficiency $50-75 \mathrm{nmol} / \mathrm{L}$; deficiency $<50 \mathrm{nmol} / \mathrm{L}$ ), folate deficiency by values below $5.3 \mathrm{ng} /$ $\mathrm{mL}$, hypovitaminosis B12 by values below $188 \mathrm{pg} / \mathrm{mL}$, hypovitaminosis $\mathrm{E}$ by vitamin $\mathrm{E}$ values below $11.6 \mu \mathrm{mol} / \mathrm{L}$, and hypovitaminosis A by vitamin A values below $1.05 \mu \mathrm{mol} / \mathrm{L}$. Copper deficiency was defined by values below $0.81 \mathrm{mg} / \mathrm{L}$, zinc deficiency by values below $0.54 \mathrm{mg} / \mathrm{L}$, and deficiency in selenium by values below $50 \mu \mathrm{g} / \mathrm{L}$. Hyperparathyroidism as a surrogate for calcium deficiency is defined by PTH values above $56.9 \mathrm{pg} / \mathrm{dL}$ and hypoferritinemia as a surrogate for iron deficiency by ferritin values below $15 \mu \mathrm{g} / \mathrm{L}$. Anemia was defined as a hemoglobin value below $12.5 \mathrm{~g} /$ $\mathrm{dL}$ in women and below $13 \mathrm{~g} / \mathrm{dL}$ in men (Table 1 ).

To investigate whether micronutrient deficiencies depend on sex or the kind of surgery, they were compared using the KruskalWallis test in the case of surgery and the Mann-Whitney $U$ test in the case of sex, as the data were not normally distributed. A $p$ value $>0.05$ was considered as statistically significant. All analyses were conducted using the SPSS 24.0 statistical software package (IBM Corp.).

\section{Results}

Out of 1,216 patients undergoing surgery $(77.3 \%$ women, mean age $41 \pm 12$ years, mean BMI $44 \pm 9$ ), 485 participated in the postoperative follow-up in the first year after surgery. The other 731 patients who underwent surgery in our clinic were already lost for follow-up at the first planned control. The mean age of the patients was 42 \pm 12 years and the mean BMI was $33.9 \pm 19.2$ at the first year after bariatric surgery; $76.9 \%$ of the patients were female. The patients were followed for 3 years. The number of patients undergoing the recommended yearly exami- nation decreased to 114 patients in year $2(\mathrm{BMI} 29.7 \pm 8.7)$ and 80 patients in year 3 (BMI 26.2 \pm 4.0$) .42 .7 \%(n=207)$ of patients attending the clinic 1 year after surgery underwent sleeve gastrectomy, $43.7 \%(n=211)$ RYGB, and $13.9 \%(n=67)$ gastric banding. In the second year of follow-up, 45 patients with sleeve gastrectomy, 54 patients with RYGB, and 15 patients with gastric banding were still available for analysis. In the third year, this number decreased to 25 patients with sleeve gastrectomy, 44 patients with RYGB, and 11 patients with gastric banding. Patient characteristics at 1,2, and 3 years of follow-up are shown in Table 2 . The baseline vitamin levels are shown in Table 1.

Overall, $29.7 \%(n=144)$ of the patients had a deficit in folic acid after the first year of surgery, decreasing to $21.6 \%$ in year 2 and $15.3 \%$ in year 3 . Regarding vitamin B12, $14.3 \%$ ( $n=69$, year 1 ) exhibited a deficit (year $2,1.8 \%$ $[n=2]$; year 3, 6.3\% [ $n=5])$. For 25-OH-vitamin D, 70.8\% $(n=343)$ showed a deficit in year 1 (year $2,67.0 \%[n=$ 76]; year 3, 57.4\% $[n=46])$ and $26.8 \%(n=130)$ exhibited elevated levels of PTH (year 2, 30.3\% [ $n=35]$; year 3, $31.1 \%[n=25])$.

Iron deficiency in iron was found as follows: $21.6 \%$ $(n=105$, year 1$), 35.0 \%(n=40$, year 2$), 32.5 \%(n=26$, year 3). Likewise, a decrease in hemoglobin was seen in $28.2 \%(n=137)$ of patients in year 1 (year $2,36.4 \%[n=$ 52]; year 3, 37.0\% [ $n=30])$. Vitamin A deficiency rates were as follows: $13.2 \%(n=64$, year 1$), 8.9 \%(n=101$, year $2), 12.8 \%(n=10$, year 3$)$. None of the patients had a deficit in vitamin $\mathrm{E}$.

Zinc deficiency was only found in year $1(1.7 \%, n=8)$ and $3(1.5 \%, n=1)$. There was a deficit in copper in $10.4 \%$ $(n=50$, year 1$), 12.2 \%(n=14$, year 2$)$, and $11.9 \%(n=10$, year 3$)$. Selenium deficit was only found in year $1(11.1 \%$, $n=54)$ and year $2(4.3 \%, n=5)$. 
Fig. 1. Prevalence of vitamin deficiency (\%) 1,2 , and 3 years after bariatric surgery.

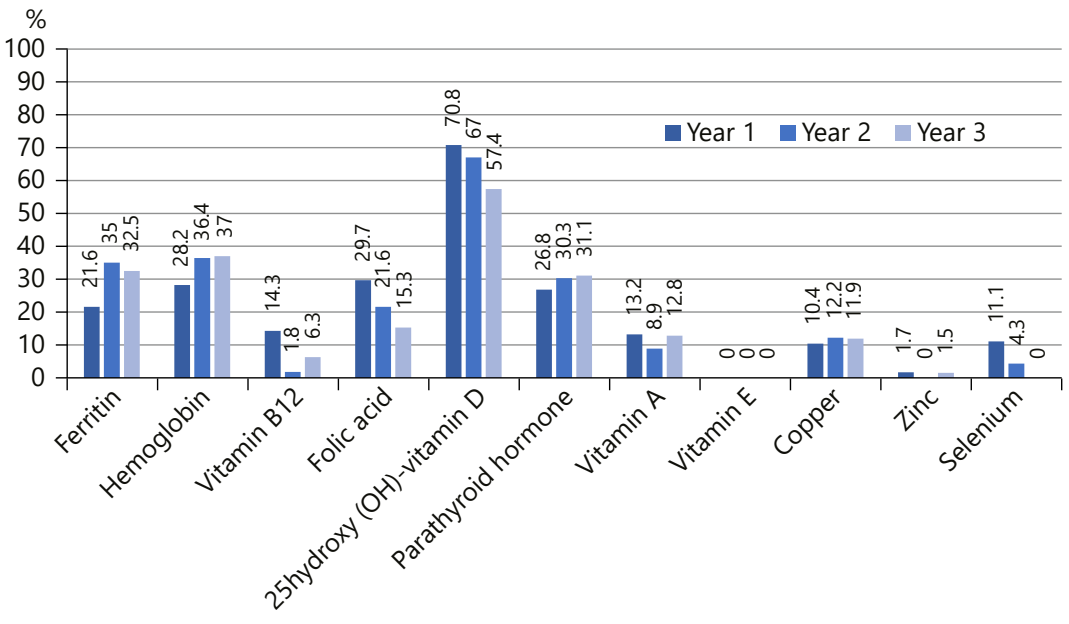

Comparing micronutrient deficiencies in patients before and after surgery there was an increase in the deficiency of ferritin, vitamin B12, vitamin A, copper, and selenium. Vitamin $\mathrm{E}$ and zinc did not show a difference and the deficiency in vitamin $\mathrm{D}$ and folic acid decreased. A summary of all deficits is provided in Figure 1 and Table 1.

Gender-specific differences were only seen in the first 2 years of follow-up. Women significantly more often exhibited low levels of hemoglobin (year 1, 32.1 vs. 9.6\%, $p<0.002$; year 2, 41.1 vs. $15.0 \%, p=0.029)$ and a deficit in in ferritin (year $1,24.6$ vs. $8.0 \%, p=0.002$; year $2,39.8$ vs. $15.8 \%, p=0.048)$.

On the other hand, deficiency in copper was seen significantly more often in men than in women (year 1, 44.4 vs. $5.4 \%, p<0.002$; year $2,66.7$ vs. $8.1 \%, p=0.004)$. In the first year of follow-up a deficiency in vitamin B12 was seen slightly more often in men ( $21.1 \mathrm{vs.} 12.8 \%, p=0.043$ ).

Considering the type of surgery, significant differences in several vitamins were seen predominantly in the first year of follow-up, especially regarding patients with sleeve gastrectomy versus RYGB. After RYGB, there were significantly more deficits in hemoglobin (36.7 vs. $18.4 \%$, $p<0.002$ ), ferritin (32.4 vs. 9.3\%, $p<0.002$ ), vitamin B12 (16.7 vs. $4.9 \%, p<0.002)$, vitamin $\mathrm{D}(90.3$ vs. $49.2 \%, p<$ $0.002)$, vitamin A (33.3 vs. 9.0\%, $p=0.017)$, and selenium ( 66.7 vs. $6.1 \%, p=0.002$ ). PTH was significantly more often elevated in RYGB (38.3 vs. $14.1 \%, p<0.002)$.

In the second year of follow-up, these differences were only seen for hemoglobin (51.8 vs. $20.8 \%, p=0.001$ ), ferritin ( 52.6 vs. $17.6 \%, p<0.002$ ), and vitamin $\mathrm{D}$ (93.9 vs. $37.0 \%, p<0.002)$, and elevation of PTH continued to persist ( 45.6 vs. $10.4 \%, p<0.002$ ). In the third year of follow- up significant differences for hemoglobin (51.2 vs. 19.4\%, $p=0.004$ ), ferritin ( 47.5 vs. $18.4 \%, p=0.007)$, PTH ( 48.7 vs. $12.1 \%, p=0.001)$, and vitamin $\mathrm{D}(78.1$ vs. $38.2 \%, p=$ $0.001)$ continued to be prevalent. There was, however, a newly observed difference in vitamin A deficiency (RYGB 31.3 vs. sleeve gastrectomy $0 \%, p=0.005$ ).

Regarding differences in sleeve gastrectomy versus gastric banding, an elevation in PTH (35.7 vs. $14.1 \%, p=$ 0.035 ) and a deficiency in folic acid ( 45.6 vs. $23.0 \%, p=$ 0.001 ), vitamin B12 (21.8 vs. $4.9 \%, p<0.002$ ), and vitamin $\mathrm{D}$ ( 87.5 vs. $49.2 \%, p=0.036)$ were found significantly more often in patients with gastric banding in the first year of follow-up. Those significant differences continued to persist during the second year for elevations in PTH (66.7 vs. $10.4 \%, p=0.035$ ) and folic acid deficiency (50.0 vs. $8.9 \%$, $p=0.001)$. A deficiency in vitamin $\mathrm{D}$ was significantly more often seen in sleeve gastrectomy than gastric banding during the second year of follow-up ( $37.0 \mathrm{vs.} 0 \%, p=0.036)$.

Regarding differences in gastric banding versus RYGB, there was only seen a significantly higher deficit in folic acid during the first 2 years of follow-up (year 1, gastric banding 45.6 vs. RYGB $30.3 \%, p=0.029$; year 2 , gastric banding $59.0 \%$ vs. RYGB $30.8 \%, p=0.029$ ). During the third year of follow-up, there were no significant differences in gastric banding versus sleeve gastrectomy or RYGB.

\section{Discussion}

Morbid obesity is accompanied by multiple health-related consequences such as diabetes mellitus and cardiovascular disease resulting in an increased burden to the 
individual and the society. Bariatric surgery provides numerous benefits such as a reduction in the prevalence of hypertension, hyperlipidemia, diabetes mellitus, sleep apnea and overall cardiovascular risk, as well as an increase in health-related quality of life [8]. It is well known, however, that bariatric surgery as an effective treatment can induce postoperative micronutrient deficiencies, which require substitution [2], especially when malabsorptive procedures are applied [9].

In our study we were able to demonstrate a high prevalence of micronutrient deficiency in patients after bariatric surgery in a large population. Weight loss after surgery was satisfactory and all patients were advised regarding continuous micronutrient substitution and follow-up visits. As shown by others [5], the frequency of postoperative visits decreased over the years. After the first year of follow-up, we could observe significantly more deficiencies in ferritin, vitamin B12, vitamin D, vitamin A, and selenium, as well as an elevation in PTH and a decrease in hemoglobin in patients following RYGB versus sleeve gastrectomy. These findings are in line with previous observations [10,11]; however, there are also contradicting reports which could not observe any differences for micronutrients [12] or, on the contrary, observed a decrease of deficiency of vitamin D [13].

These differences can be explained by the respective surgical technique. In sleeve gastrectomy, only the large curvature of the stomach is removed, while the anatomy of the gastrointestinal tract remains intact. However, the reduced availability of stomach secretion of pepsin and hydrochloric acid may per se lead to a decreased capacity for digestion [14]. In RYGB, the stomach is reduced to a volume of $20-30 \mathrm{~mL}$ and the stomach contents are rerouted to the distal jejunum via an anastomosis connection. Therefore, the duodenum and proximal jejunum the main parts of micronutrient absorption - are excluded from resorption.

The highest deficit was found in vitamin $\mathrm{D}$, which is congruent with previously published preoperative data [15]. Therefore, it can be assumed that a preoperative deficit is difficult to compensate and continues to persist postoperatively despite intensified vitamin substitution. Nevertheless, in our study $>70 \%$ of patients exhibited a deficiency of vitamin $\mathrm{D}$. In the long-term follow-up, the deficit in vitamin $\mathrm{D}$ decreased to below $60 \%$ as a consequence of intensive patient counseling and substitution, which is in line with published observations [13]. Lower 25-OH-vitamin D levels are associated with metabolic bone disease and osteoporosis and, as recently shown, advanced stages of liver fibrosis in morbidly obese patients

Micronutrient Deficiency after Bariatric

Surgery
[16]. Since 25-OH- vitamin D is processed through sunlight, it has a significant relationship to physical activity [17].

PTH is an important regulator of calcium homeostasis and thus of bone metabolism. We consequently found elevated levels of PTH indicating secondary hyperparathyroidism caused by concurrent vitamin D deficiency in nearly $27 \%$ of our patients 1 year after surgery. The respective levels were slightly rising after 2 and 3 years.

Folic acid is characterized by a low storage capacity. The main resorption sites are the duodenum and proximal jejunum. Folic acid deficiency might lead to megaloblastic pernicious anemia and neural tube defects [18]. A deficit in folic acid was shown in $30 \%$ of patients and decreased continuously during the follow-up. In comparison to preoperative data, the number of patients with a deficiency was much lower [15], most likely due to intensive substitution already started preoperatively. Indeed, the levels of folic acid were higher than those reported by others $[11,19]$.

The main site of absorption of vitamin B12 is the terminal ileum. The storage capacity of vitamin B12 in the body is quite large; however, vitamin B12 deficiency can lead to hematologic and neurologic symptoms. In particular, macrocytic anemia following bariatric surgery may indicate vitamin B12 deficiency. Vitamin B12 deficiency was prevalent in $14 \%$ of the study population and decreased significantly over time due to intramuscular administration of vitamin B12 every 3 months, especially in RYGB.

The main sites of absorption for iron are the duodenum and the proximal jejunum. Common physical symptoms of iron deficit are fatigue, reduced exercise performance, and anemia. A deficit in ferritin as a surrogate for iron deficiency was shown in $22 \%$ of the patients and further increased during follow-up. Anemia was seen in 32\% of women and nearly $10 \%$ of men after the first year of surgery. This gender difference is most likely caused by an iron loss due to menstruation. These numbers increased during follow-up in both sexes. Deficits in ferritin were seen significantly more often in RYGB than sleeve gastrectomy, which is in line with other reports [20,21].

Fat-soluble vitamin A is mainly resorbed in the upper small intestine. Normally, vitamin A deficiency is most likely seen in third-world countries and in people with defects in liver storage. Common symptoms of vitamin A deficiency are night blindness, reduced infection control, and problems in reproduction [22,23]. A deficit in vitamin A was seen in $13 \%$ of the examined patients in the first postoperative year and did not change after 3 years. 
Other authors likewise demonstrated that vitamin A deficiency was relatively common after bariatric surgery $[10,24]$.

Vitamin $\mathrm{E}$ is an important antioxidant. As there is sufficient liver storage capacity, vitamin E deficiency is only seen after many years of malnutrition. Accordingly, no patient in our study exhibited vitamin E deficiency.

Copper is essential for the pigmentation of skin and hair and indirectly influences blood building through influencing iron. Furthermore, it plays a role in the proper function of the immune system. The main resorption sites are the stomach and duodenum. Around 10\% of our patients exhibited a deficit in copper. This number increased slightly to $12 \%$ in the third year of follow-up as also reported in the literature [25].

Selenium is an essential cofactor of selenoproteins. The main sites of absorption are the duodenum and proximal jejunum. Common symptoms of selenium deficit are myopathy, cardiomyopathy, and loss of muscle mass. In our population, $11 \%$ of patients exhibited a selenium deficit during the first year of follow-up. This level decreased continuously, and after 3 years of follow-up we could not find a selenium deficit anymore. Until now, there have only been a few studies examining selenium levels after bariatric surgery as it is not recommended as a routine examination by the ASMBS guidelines [26]. Our results are also in line with published data [25].

Zinc is a trace element essential for wound healing, growth, and reproductive function. The main site of absorption is the jejunum and the storage capacity of the body is quite small. Typical symptoms of a zinc deficit are skin rashes, acne, decrease in taste, increased susceptibility to infection and, in severe zinc deficiency, also hypogonadism, alopecia, deficits in wound healing, and diarrhea. A deficit in zinc was found in only $2 \%$ of the study population, which is in line with another study [25], but significantly less common than found by other authors $[27,28]$.

In our study we found some significant gender differences. Women more often exhibited a decrease in hemoglobin and a deficit in ferritin. A deficit in ferritin is caused either by inadequate intake or absorption of iron, or blood loss. The higher amount of deficiency in women may be due to blood loss caused by menstrual bleeding. Similar results were shown by other authors [29]. This finding shows once again the importance of developing and following a standard protocol for monitoring postoperative patients, especially women of childbearing age. Men more often showed a deficit in vitamin B12 during the first year of follow-up and a deficit in copper during the first 2 years.

When comparing the effect of both surgical methods, sleeve gastrectomy proved to pose less risk for postoperative micronutrient deficiencies. Most common were deficiencies in vitamin $\mathrm{D}$ and folic acid, which have also been prevalent already preoperatively [15] and which are also found in the general population [30,31]. There was a quite high number of deficiencies found in gastric banding compared to sleeve gastrectomy despite the fact that both are solely restrictive procedures. However, the restriction in gastric banding could be more pronounced due to a smaller pouch and a narrower passage depending on the filling of the band. This might lead to altered eating habits, thereby possibly avoiding high-volume food rich in vitamins and micronutrients. The drop-out rate in year 3 was similar in patients with sleeve gastrectomy and gastric banding.

Our findings stress the importance of a thorough postoperative assessment of micronutrient status as well as of reinforcement of scheduled visits. Unfortunately, specific postoperative laboratory controls are performed in $<50 \%$ of patients after bariatric surgery, depending on the kind of vitamin or micronutrient investigated, as shown by Gudzune et al. [5]. Since the majority of patients completing the follow-up after 1 year were lost over time, we have to assume that the true micronutrient prevalence might be higher in the entire population.

One strength of our study is the high number of subjects investigated. The higher number of female patients reflects the observation that women more often undergo bariatric surgery than men. A limitation to be mentioned is the given seasonal variation of vitamin $\mathrm{D}$ in Austria due to differences in sun exposure. Since we determined vitamin $\mathrm{D}$ throughout the year, bias regarding seasonal variation should be minimized. The decreased number of patients showing up for follow-up over the years is another limitation but a common phenomenon. We cannot distinguish, however, whether patients with postoperative complications are more likely to seek contact to physicians specialized in the treatment of bariatric patients or whether patients being more adherent in general are more likely to undergo the recommended postoperative check-ups. Therefore, the system of follow-up needs to be standardized and reinforced. In addition, no formal drug accountability was performed as it was not feasible in such a large patient population. The patients, however, were asked at each visit about their intake of the respective supplements and reminded to continue taking them permanently. 
In conclusion, our data emphasize the importance of a stringent postoperative evaluation regarding nutritional deficiencies in addition to postoperative surveillance and respective substitution. Awareness should be raised in all physicians that after bariatric surgery subjects might be at risk for clinically important nutritional deficiencies and should be encouraged to attend regular follow-up visits.

\section{Acknowledgements}

The authors thank all patients who participated in this study and medical staff of the Clinic Landstrasse who helped with the data collection.

\section{Statement of Ethics}

This research was conducted ethically in accordance with the World Medical Association Declaration of Helsinki. Subjects gave written informed consent before data collection and the study protocol was approved by the Ethics Committee of Vienna (Magistrat der Stadt Wien MA 15; EK 19-077-VK).

\section{Conflict of Interest Statement}

No potential conflicts of interest relevant to this article were reported. During the last 3 years E.-C.K. received honoraria for national lectures by the Austrian Obesity Society. J.M.B. received honoraria from AstraZeneca, Boehringer Ingelheim, Eli Lilly, MSD, Novo Nordisk, and Sanofi aventis. B.L. received grant support and honoraria for research projects and advice from Eli Lilly, Novo Nordisk, Sanofi, Boehringer Ingelheim, AstraZeneca, Bayer, Amgen. A.S. and V.P. have nothing to disclose.

\section{Funding Sources}

There was no funding for this research.

\section{Author Contributions}

E.-C.K. performed the calculations, analyzed the data, and wrote the manuscript; A.S. helped to collect the data; J.M.B. helped analyzing the data; J.M.B. and V.P. provided critical feedback; B.L. supervised the findings of this work. All authors contributed to the final manuscript.

\section{References}

1 World Health Organisation. Obesity and overweight: key facts [cited 2020 May 24]. Available form: www.who.int/mediacentre/ factsheets/fs311/en/.

2 Stein J, Stier C, Raab H, Weiner R. Review article: the nutritional and pharmacological consequences of obesity surgery. Aliment Pharmacol Ther. 2014 Sep;40(6):582-609.

3 Ben-Porat T, Elazary R, Goldenshluger A, Sherf Dagan S, Mintz Y, Weiss R. Nutritional deficiencies four years after laparoscopic sleeve gastrectomy-are supplements required for a lifetime? Surg Obes Relat Dis. 2017 Jul;13(7):1138-44.

4 Caron M, Hould FS, Lescelleur O, Marceau S, Lebel S, Julien F, et al. Long-term nutritional impact of sleeve gastrectomy. Surg Obes Relat Dis. 2017 Oct;13(10):1664-73.

5 Gudzune KA, Huizinga MM, Chang HY, Asamoah V, Gadgil M, Clark JM. Screening and diagnosis of micronutrient deficiencies before and after bariatric surgery. Obes Surg. 2013 Oct;23(10):1581-9.

6 Thereaux J, Lesuffleur T, Païta M, Czernichow S, Basdevant A, Msika S, et al. Long-term follow-up after bariatric surgery in a national cohort. Br J Surg. 2017 Sep;104(10):1362-71.

7 World Medical Association. WMA Declaration of Helsinki - ethical principles for medi$\mathrm{cal}$ research involving human subjects [cited 2020 March 16]. Available from: https://www. wma.net/policies-post/wma-declaration-ofhelsinki-ethical-principles-for-medical-research-involving-human-subjects/.
8 Sjöström L. Review of the key results from the Swedish Obese Subjects (SOS) trial - a prospective controlled intervention study of bariatric surgery. J Intern Med. 2013 Mar;273(3): 219-34.

9 Maggard MA, Shugarman LR, Suttorp M, Maglione M, Sugerman HJ, Livingston EH, et al. Meta-analysis: surgical treatment of obesity. Ann Intern Med. 2005 Apr;142(7):547-59.

10 Johnson LM, Ikramuddin S, Leslie DB, Slusarek B, Killeen AA. Analysis of vitamin levels and deficiencies in bariatric surgery patients: a single-institutional analysis. Surg Obes Relat Dis. 2019 Jul;15(7):1146-52.

11 Gehrer S, Kern B, Peters T, Christoffel-Courtin C, Peterli R. Fewer nutrient deficiencies after laparoscopic sleeve gastrectomy (LSG) than after laparoscopic Roux-Y-gastric bypass (LRYGB)-a prospective study. Obes Surg. 2010 Apr;20(4):447-53.

12 Moizé V, Andreu A, Flores L, Torres F, Ibarzabal A, Delgado S, et al. Long-term dietary intake and nutritional deficiencies following sleeve gastrectomy or Roux-En-Y gastric bypass in a mediterranean population. J Acad Nutr Diet. 2013 Mar;113(3):400-10.

13 Fox A, Slater C, Ahmed B, Ammori BJ, Senapati S, Akhtar K, et al. Vitamin D Status After Gastric Bypass or Sleeve Gastrectomy over 4 Years of Follow-up. Obes Surg. 2020 Apr; 30(4):1473-81.

14 Behrns KE, Smith CD, Sarr MG. Prospective evaluation of gastric acid secretion and cobal- amin absorption following gastric bypass for clinically severe obesity. Dig Dis Sci. 1994 Feb; 39(2):315-20.

15 Krzizek EC, Brix JM, Herz CT, Kopp HP, Schernthaner GH, Schernthaner G, et al. Prevalence of Micronutrient Deficiency in Patients with Morbid Obesity Before Bariatric Surgery. Obes Surg. 2018 Mar;28(3):643-8.

16 Luger M, Kruschitz R, Kienbacher C, Traussnigg S, Langer FB, Schindler K, et al. Prevalence of liver fibrosis and its association with non-invasive fibrosis and metabolic markers in morbidly obese patients with vitamin D deficiency. Obes Surg. 2016 Oct;26(10):242532.

17 Holick MF. Vitamin D deficiency. N Engl J Med. 2007 Jul;357(3):266-81.

18 Milunsky A, Jick H, Jick SS, Bruell CL, MacLaughlin DS, Rothman KJ, et al. Multivita$\mathrm{min} /$ folic acid supplementation in early pregnancy reduces the prevalence of neural tube defects. JAMA. 1989 Nov;262(20):2847-52.

19 Pellitero S, Martínez E, Puig R, Leis A, Zavala R, Granada ML, et al. Evaluation of Vitamin and Trace Element Requirements after Sleeve Gastrectomy at Long Term. Obes Surg. 2017 Jul;27(7):1674-82.

20 Ferraz ÁA, Carvalho MR, Siqueira LT, SantaCruz F, Campos JM. Micronutrient deficiencies following bariatric surgery: a comparative analysis between sleeve gastrectomy and Roux-en-Y gastric bypass. Rev Col Bras Cir. 2018 Dec;45(6):e2016. 
21 Gu L, Fu R, Chen P, Du N, Chen S, Mao D, et al. In Terms of Nutrition, the Most Suitable Method for Bariatric Surgery: Laparoscopic Sleeve Gastrectomy or Roux-en-Y Gastric Bypass? A Systematic Review and Meta-analysis. Obes Surg. 2020 May;30(5):2003-14.

22 Lee WB, Hamilton SM, Harris JP, Schwab IR. Ocular complications of hypovitaminosis a after bariatric surgery. Ophthalmology. 2005 Jun;112(6):1031-4.

23 Cañete A, Cano E, Muñoz-Chápuli R, Carmona R. Role of vitamin A/retinoic acid in regulation of embryonic and adult hematopoiesis. Nutrients. 2017 Feb;9(2):159.

24 Zalesin KC, Miller WM, Franklin B, Mudugal D, Rao Buragadda A, Boura J, et al. Vitamin a deficiency after gastric bypass surgery: an underreported postoperative complication. J Obes. 2011;2011:760695.
25 Papamargaritis D, Aasheim ET, Sampson B, le Roux CW. Copper, selenium and zinc levels after bariatric surgery in patients recommended to take multivitamin-mineral supplementation. J Trace Elem Med Biol. 2015; 31:167-72.

26 Parrott J, Frank L, Rabena R, Craggs-Dino L, Isom KA, Greiman L. American Society for Metabolic and Bariatric Surgery Integrated Health Nutritional Guidelines for the Surgical Weight Loss Patient 2016 Update: micronutrients. Surg Obes Relat Dis. 2017 May;13(5): $727-41$.

27 Ruz M, Carrasco F, Rojas P, Codoceo J, Inostroza J, Basfi-fer K, et al. Zinc absorption and zinc status are reduced after Roux-en-Y gastric bypass: a randomized study using 2 supplements. Am J Clin Nutr. 2011 Oct;94(4): 1004-11.
28 Sallé A, Demarsy D, Poirier AL, Lelièvre B, Topart P, Guilloteau G, et al. Zinc deficiency: a frequent and underestimated complication after bariatric surgery. Obes Surg. 2010 Dec; 20(12):1660-70.

29 Engebretsen KV, Blom-Høgestøl IK, Hewitt S, Risstad H, Moum B, Kristinsson JA, et al. Anemia following Roux-en-Y gastric bypass for morbid obesity; a 5-year follow-up study. Scand J Gastroenterol. 2018 Aug;53(8):91722.

30 Holick MF. The vitamin D deficiency pandemic: approaches for diagnosis, treatment and prevention. Rev Endocr Metab Disord. 2017 Jun;18(2):153-65.

31 Rogers LM, Cordero AM, Pfeiffer CM, Hausman DB, Tsang BL, De-Regil LM, et al. Global folate status in women of reproductive age: a systematic review with emphasis on methodological issues. Ann NY Acad Sci. 2018 Nov;1431(1):35-57. 